\title{
«Wir sind nicht nur im Fussball gut, wir sind auch im Gesundheitswesen Weltspitze»
}

\author{
Weg von der monotonen Kostendiskussion, hin zu einer ganzheitlichen Betrachtung: \\ Das war der ambitionierte Anspruch des Sessionsanlasses der FMH vom 16. Juni in \\ Bern zum Thema «Volkswirtschaftliche Bedeutung des Gesundheitswesens». Bundes- \\ präsidentin Doris Leuthard sowie weitere namhafte Referentinnen und Referenten \\ sollten für einmal den Fokus auf das Potential und den Nutzen des Gesundheits- \\ wesens als eine der tragenden Säulen unserer Volkswirtschaft richten.
}

Hupende Autos, Sprechchöre und Fangesänge: Nach dem eben Tatsache gewordenen, historischen Sieg der Schweizer Fussballer über den amtierenden Europameister und WM-Mitfavoriten Spanien schwappten die Wellen der Begeisterung am Abend des 16. Juni aus den Gassen Berns auch immer wieder in den ehrwürdigen Saal des Hauses Zum Äusseren Stand hinein. Eine Vorlage, die von einzelnen Referenten dankbar und nicht ohne rhetorisches Geschick aufgenommen wurde - etwa von Peter Kappert: «Wir sind nicht nur im Fussball gut, wir sind auch im Gesundheitswesen Weltspitze», eröffnete der Präsident der «Swiss Leading Hospitals» und Mitglied des Beirats von «Swiss Health» sein Referat.

\section{Doris Leuthard: wegkommen von der unseligen Kostendiskussion}

Tatsächlich waren am Sessionsanlass der FMH positive Äusserungen zum schweizerischen Gesundheitswesen in einer Dichte zu hören, die bei gesundheitspolitischen und -ökonomischen Veranstaltungen Seltenheitswert hat. Dass dies durchaus in der Absicht der Organisatoren lag, brachte FMH-Präsident Jacques de Haller als Gastgeber bereits in seinem Begrüssungswort zum Ausdruck. «Das Gesundheitswesen soll für einmal aus einem anderen Gesichtswinkel betrachtet werden», umriss er eine zentrale Idee des Abends. «Wir wollen uns von der Frage leiten lassen, was das Gesundheitswesen als wirtschaftlich bedeuten-
Korrespondenz: Dr. med. et lic. phil. Bruno Kesseli EMH Farnsburgerstrasse 8 CH-4132 Muttenz

bkesseli@emh.ch

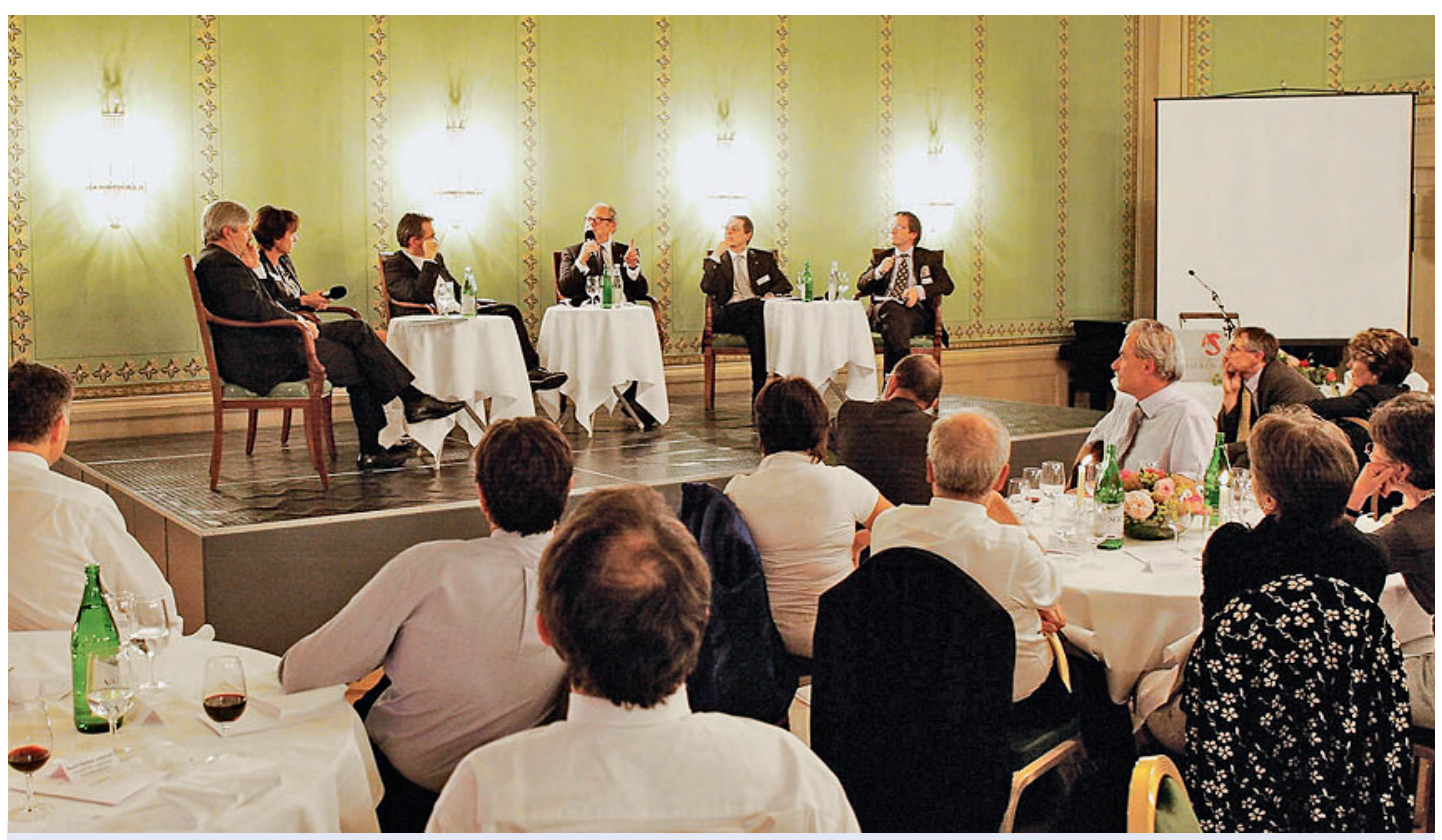

Der fehlende Nachwuchs - sowohl in der Ärzteschaft als auch in der Pflege - war eines der Themen der Podiumsdiskussion. 


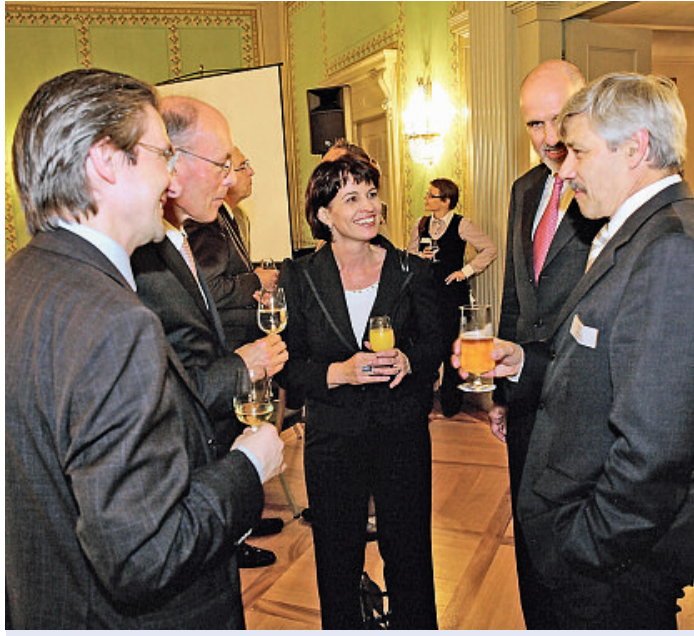

Angeregter Austausch.

der, wachsender Sektor der Gesellschaft bringen kann.»

Doris Leuthard, Bundespräsidentin und Vorsteherin des Eidgenössischen Volkswirtschaftsdepartements, unterstützte diese Stossrichtung mit einem wahren Feuerwerk aus Zahlen und Fakten. Rund 12\% der arbeitstätigen Schweizer Bevölkerung sind im Gesund-
Rang 4 ein. Nicht zu vernachlässigen sind auch die Forschungsinvestitionen von 500-600 Millionen Franken pro Jahr.

Die Volkswirtschaftsministerin betonte indes auch, dass sich das Gesundheitswesen mit seinem Wertschöpfungseffekt von rund 60 Milliarden Franken aus zwei völlig unterschiedlichen Bereichen zusammensetze. Neben der Produktionsbranche gehöre dazu auch der Dienstleistungssektor mit seiner unterdurchschnittlichen Produktivität. Dass dem so sei, liege gewissermassen in der Natur der Sache: «Pflege kann nicht wertschöpfend sein»- zudem fielen in diesem Bereich hohe administrative Kosten an. Dennoch: Die Arbeitsprozesse, so Leuthard, seien im Dienstleistungssektor generell noch zuwenig effizient. «Nach volkswirtschaftlichen Massstäben müsste hier angesetzt werden.» Als besorgniserregend stufte sie ein, dass die Gesamtausgaben für das Schweizer Gesundheitswesen in den letzten 40 Jahren von 5,4\% des Bruttoinlandproduktes (BIP) auf aktuell 11\% gestiegen sind. «Dieser Trend ist ungebrochen, und das kann uns nicht gefallen.»

In einer Art Synthese plädierte Leuthard zum Schluss dafür, den «Wirtschaftsteil Gesundheitswesen» nicht übermässig zu segmentieren, sondern «aus einer Gesamtsicht» zu betrachten. «Dann kämen wir auch von der unseligen Kostendiskussion weg.» Mit einer

\section{«Es ist Zeit, dass sich auch die Politik der Bedeutung und des Werts unseres Gesundheitswesens als Gesamtes bewusst wird» Jacques de Haller}

heitssektor beschäftigt, wobei das Beschäftigungswachstum in den letzten Jahren mit 10\% gegenüber $7 \%$ in den übrigen Branchen eindrücklich ist. Allein im Pharmasektor arbeiten 120000 Menschen, dazu kommen 40000 im Bereich der Medizinaltechnik. Was die Anzahl Beschäftigter betrifft, nimmt das MedtechSegment, das mit 8-10\% stolze jährliche Wachstumsraten aufweist, im europäischen Vergleich immerhin solchen Betrachtungsweise, gab sich die Bundespräsidentin überzeugt, könnten «Blockierungen gelöst werden und ein starker Pfeiler entstehen, der den Standort Schweiz stärkt».

Die Aussagen über mangelnde Effizienz schienen bei einem Teil der Hörerschaft stärker haften geblieben zu sein als die «Gesamtsicht». Dies liess zumindest der letzte Teil der Veranstaltung vermuten, in dem

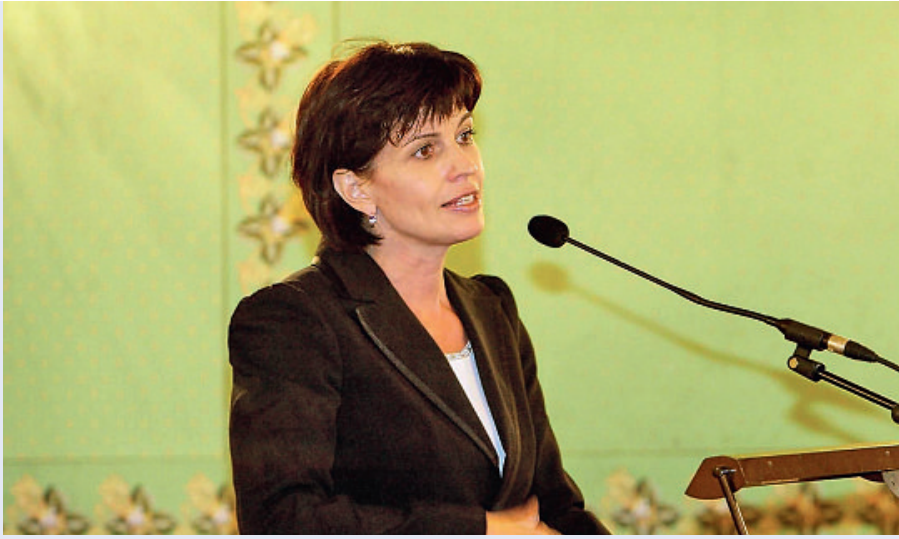

Für Bundespräsidentin Doris Leuthard ist das Schweizer Gesundheitswesen auch ein wachsender Wirtschaftszweig.

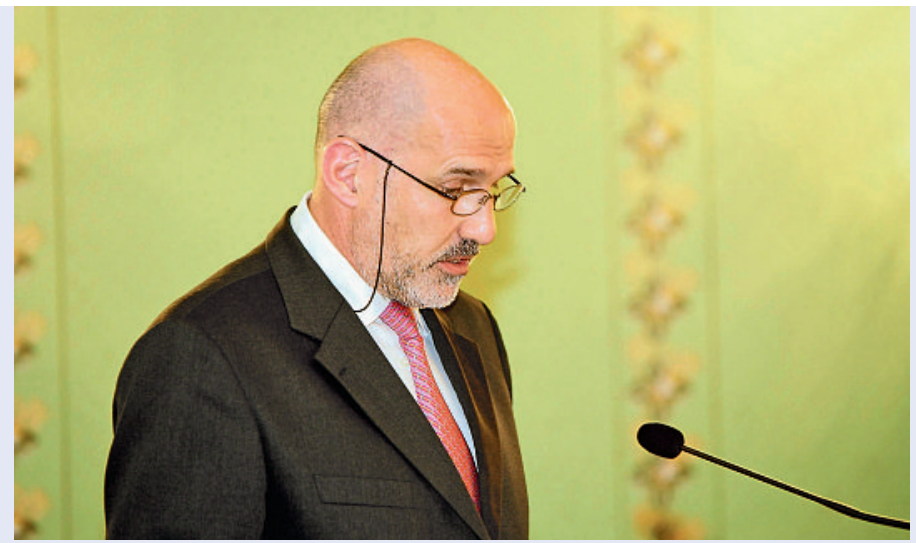

Für Jacques de Haller steht das Wohl der Patienten an erster Stelle. 


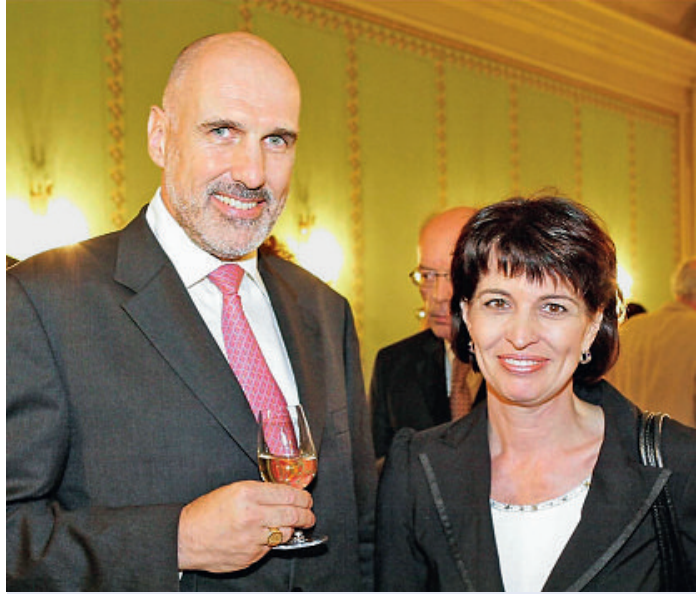

Bundespräsidentin Doris Leuthard und FMH-Präsident Jacques de Haller.

das Publikum zu Wort kam. «Ich nehme hier zu wenig Freude und Stolz wahr», meinte etwa Peter Wiedersheim, St. Galler Internist und Rheumatologe sowie profilierter Standespolitiker. «Wir hauen uns Kosten und Effizienz um die Ohren, vergessen dabei aber die volkswirtschaftliche Gesamtbetrachtung. Ich fühle mich in diesem System nur noch als «eilungskostenverursacher > die wertvolle Leistung, die ich gelegentlich doch auch zu erbringen meine, geht völlig unter.» Der spontane Applaus, den der Präsident der Konferenz der Ostschweizer Ärztegesellschaften für sein Votum erntete, liess vermuten, dass er etlichen Anwesenden aus dem Herzen gesprochen hatte.

Auch Jacques de Haller, dessen Referat unmittelbar an die Ausführungen von Bundespräsidentin Leuthard anschloss, gab ein engagiertes Plädoyer für ein Gesundheitswesen $a b$, das die Patientenbedürfnisse in den Mittelpunkt stellt und sich nicht durch ökonomische Zwänge einschnüren lässt: «Es muss gespart werden. Die Wirtschaftlichkeit muss gefördert werden. Die Effizienz muss gefördert werden. Doch nicht auf Kosten der Ärzte und schon gar nicht auf Kosten des Patientenwohls. Die Schmerzgrenze ist erreicht!» Auf die Gretchenfrage «Was ist uns die Gesundheit wert?» gab der Präsident der FMH eine unmissverständliche Antwort: «Sehr viel - lautet die Antwort der Betroffenen, 〈sehr viel - so muss auch die Antwort unserer Gesellschaft lauten.» Er schloss seine Ausführungen mit einem Appell an die Politiker: «Es ist Zeit, dass sich auch die Politik der Bedeutung und des Werts unseres Gesundheitswesens als Gesamtes bewusst wird.»

\section{Sparen kann auch kontraproduktiv sein}

Dass eine primär auf Kostenvermeidung und Sparen ausgerichtete Strategie in einer Gesamtbetrachtung keineswegs der überzeugendste Ansatz sein muss, konnten die Referenten aus Verwaltung und Industrie in ihren Vorträgen mitunter eindrücklich plausibel machen. So präsentierte Urs Birchler, Chief Executive Officer des Berner Inselspitals, eine Studie, die für den Standort Bern anhand eines spezifischen Indikatorenrasters Kosten und Nutzen eines Universitätsspitals und eines Kantonsspitals verglich. Fazit: Ein Universitätsspital belastet die Volkswirtschaft zwar stärker als ein Kantonsspital, der volkswirtschaftliche Nutzen ist aber überproportional.

\section{«Helfen Sie mit, Swissness in die}

Welt hinauszutragen» Peter Kappert

Mit den Belangen des Marketings nicht unvertraut scheint Peter Kappert. Der Präsident der «Swiss Leading Hospitals» punktete nicht nur mit dem eingangs wiedergegebenen Zitat zu den Parallelen von Fussball und Gesundheitswesen made in Switzerland. Ebenso eingängig waren Formulierungen wie «Swissness to the world» oder «Swiss houses abroad for healthcare», mit denen er seine Vision des Exports von Gesundheitsdienstleistungen und Know-how in Zielmärkte wie Osteuropa, aber auch den Mittleren und Fernen Osten umriss.

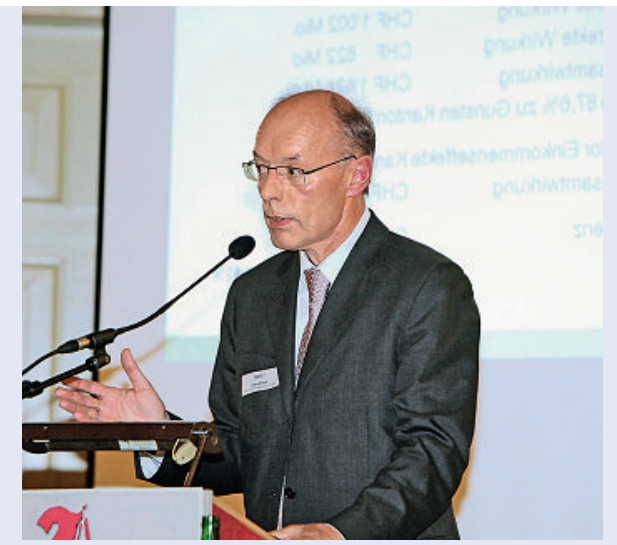

Urs Birchler,

CEO des Inselspitals Bern.

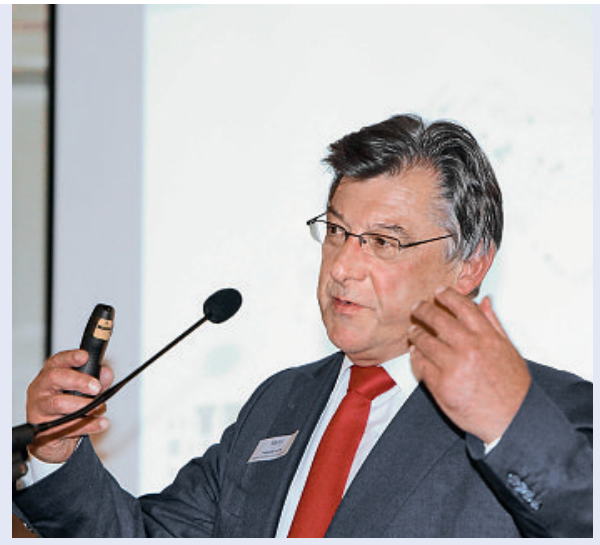

Peter Kappert,

Präsident der «Swiss Leading Hospitals».

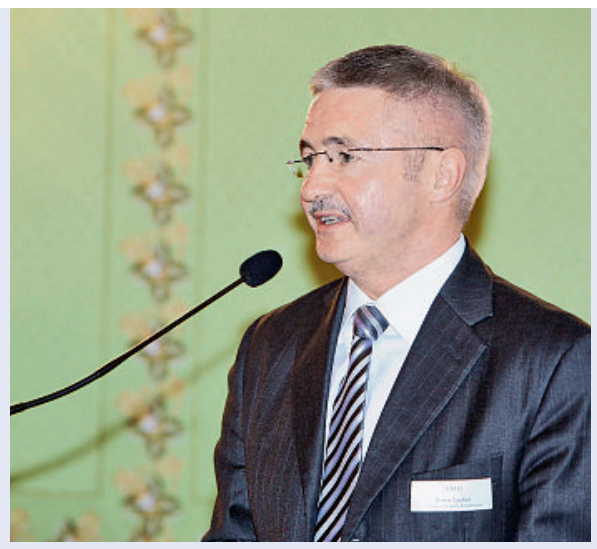

Erwin Locher,

Präsident von Medtech Schweiz. 
Umgekehrt soll Patienten in einer globalisierten Gesundheitswelt ermöglicht werden, medizinische Dienstleistungen in der Schweiz komfortabel in Anspruch zu nehmen. Um diese Ideen voranzutreiben, wurde «Swiss Health» gegründet, die «offizielle Exportplattform des Schweizer Gesundheitswesens», die verschiedenste Akteure aus dem Gesundheitswesen wie Spitäler, Medtech- und Pharmaunternehmen, aber auch Zulieferer und Hotels vereinen soll. «Helfen Sie mit, Swissness in die Welt hinauszutragen», appellierte Kappert an die anwesenden Key Players der Branche.

\section{«Wir müssen dringend damit aufhören, alle Berufe}

\section{zu akademisieren» Carlo Conti}

Über eine eigene Exportplattform verfügt auch die Medtech-Branche. Deren Präsident, Erwin Locher, schilderte anschaulich die historischen Wurzeln, aus denen der Wettbewerbsvorteil für Unternehmen der Medizinaltechnik in der Schweiz erwuchs. Etliche Firmen verdanken ihre Entstehung der Nähe zur Uhrenindustrie - günstig wirkte sich auch der Industrie- und Wissenschaftscluster mit verwandten Technologien wie Biomechanik, Materialwissenschaften oder Mikroelektronik aus. Als weitere befruchtende Faktoren führte er «Entrepreneurship» und qualifizierte Arbeitskräfte an. Daraus resultierte gemäss Locher ein blühender Wirtschaftszweig «mit einem Umsatz von über 20 Milliarden Franken und einem BIP-Anteil von 2\%, in seiner Bedeutung vergleichbar der pharmazeutischen oder der Nahrungsmittelindustrie».

\section{Animiertes Podium}

Für die abschliessende Podiumsdiskussion hatte Moderator Artur K. Vogel, Chefredaktor der Berner Tageszeitung «Der Bund», zwei Themenkreise ins Auge gefasst: «Gesundheit als volkswirtschaftlicher Faktor und Ex-

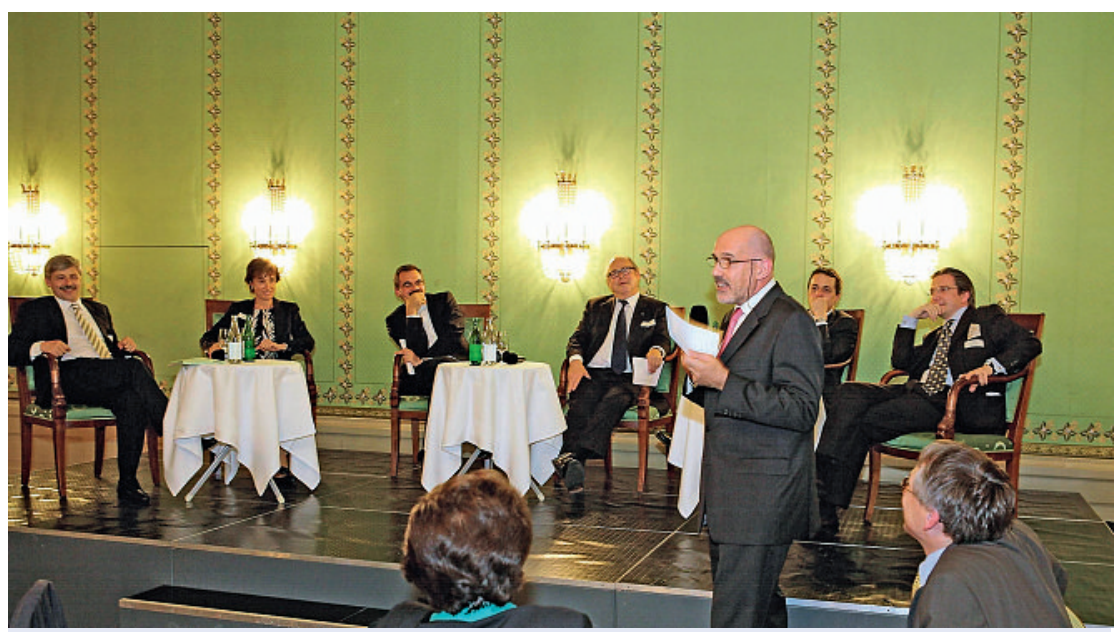

Jacques de Haller verabschiedete die zahlreich erschienenen Gäste. portgut» sowie die «Aufrechterhaltung der volkswirtschaftlich wichtigen «Branche Gesundheitswesen` angesichts des absehbaren Personalmangels». «In der zur Verfügung stehenden Zeit eine grosse Aufgabe», wie Vogel selbst einräumte. Die Podiumsgäste - Nationalrätin Ruth Humbel, ihre Kollegen Ignazio Cassis (auch FMH-Vizepräsident) und Jean-François Steiert, Gesundheitsökonom Willy Oggier sowie Carlo Conti, Vizepräsident der Gesundheitsdirektorenkonferenz - schlugen sich wacker. Für bahnbrechende Würfe und Erkenntnisse reichte es in der Tat nicht, immerhin aber für einige interessante Überlegungen und rhetorische Funken.

Dass Jean-François Steiert, der sich zeitweise auf dem Podium wie der «letzte Cartesianer» fühlte, eine andere Wahrnehmung des Gesundheitswesens hat als die auf Marktkräfte setzenden Willy Oggier und Ignazio Cassis, war keine Überraschung. Cassis zeigte sich optimistisch, dass soziale Umwelt und Markt mit adäquaten Antworten auf die sich abzeichnenden Probleme im Gesundheitswesen reagieren würden. Oggier ging gar so weit, die vielfach prognostizierte Personalknappheit im Gesundheitswesen in Zweifel zu ziehen, da er den entsprechenden Untersuchungen nur bedingt traut. Steiert widersprach - «der Mangel an medizinischen Grundversorgern beispielsweise ist bereits offensichtlich; in gewissen Regionen fehlen sie schlicht und einfach»-, aber selbst der energische Widerspruch von Bundespräsidentin Leuthard vermochte Oggier in seinen Überzeugungen nicht zu erschüttern. Im übrigen plädierte er dafür, sich nicht zu stark auf eine Personaldiskussion zu konzentrieren, sondern den Patientennutzen ins Zentrum der Überlegungen zu stellen.

Weitgehende Zustimmung fand der rhetorisch wie stets überzeugende Carlo Conti mit seiner Forderung, im Hinblick auf den - eben doch - absehbaren Personalmangel das duale Bildungssystem zu forcieren. «Wir müssen dringend damit aufhören, alle Berufe zu akademisieren, genauso, wie gewisse Berufsgruppen damit aufhören müssen, ihre Berufsbilder schlechtzureden.» Ruth Humbel stimmte ihm zu und brachte ihrerseits eine zusätzliche Idee ein. «Heute zahlen im Gesundheitswesen die Jungen für die Alten. Die ‘rüstigen Rentner könnten vermehrt ins System einbezogen werden. Man sollte darüber nachdenken, ob sich für diese 3. Generation nicht eine Art Zivildienst einführen liesse, damit die 4. Generation der Hochbetagten weiterhin gut versorgt werden kann.»

Auch wenn sich keine auf «harten Daten» basierende Gesamtbilanz des Abends ziehen lässt: Die FMH hat mit diesem lebhaften Sessionsanlass als aktiver Player der gesundheitspolitischen Diskussion konstruktive Impulse verliehen. Und sie hat den unabdingbaren direkten Austausch zwischen zentralen Akteuren des Gesundheitswesens in einer Weise gefördert, die den Boden bereitet für eine weitere partnerschaftliche Zusammenarbeit, von der im besten Fall die gesamte Bevölkerung unseres Landes profitiert. 